\title{
Frameless
}

Volume 1 | Issue 1

Article 19

November 2019

\section{Transverse Chromatic Aberrations in Virtual Reality Devices}

\author{
Ryan Beams \\ Food and Drug Administration, rbeams@optics.rochester.edu \\ Wei-Chung Cheng \\ Food and Drug Administration, wei-chung.cheng@fda.hhs.gov \\ Andrea S. Kim \\ Food and Drug Administration, andrea@mikzabiomed.com \\ Aldo Badano \\ Food and Drug Administration, aldo.badano@fda.hhs.gov
}

Follow this and additional works at: https://scholarworks.rit.edu/frameless

\section{Recommended Citation}

Beams, Ryan; Cheng, Wei-Chung; Kim, Andrea S.; and Badano, Aldo (2019) "Transverse Chromatic Aberrations in Virtual Reality Devices," Frameless: Vol. 1: Iss. 1, Article 19.

DOI: 10.14448/Frameless.01.006

Available at: https://scholarworks.rit.edu/frameless/vol1/iss1/19

This Article is brought to you for free and open access by RIT Scholar Works. It has been accepted for inclusion in Frameless by an authorized editor of RIT Scholar Works. For more information, please contact ritscholarworks@rit.edu. 


\section{FRAMELESS}

\section{Transverse Chromatic Aberrations in Virtual Reality Devices}

Ryan Beams

Center for Devices and Radiological Health Food and Drug Administration

Andrea S. Kim

Center for Devices and Radiological Health Food and Drug Administration

\section{Wei-Chung Cheng}

Center for Devices and Radiological Health

Food and Drug Administration

Aldo Badano

Center for Devices and Radiological Health

Food and Drug Administration

Abstract: We demonstrate a method for measuring the transverse chromatic aberration (TCA) in a virtual reality head-mounted display (VR HMD). This procedure was used to characterize the optical performance of the Oculus Go VR HMD. Results show a measurable TCA for angles larger than approximately $6^{\circ}$ from the center of the field of view. TCA can be thought of as a wavelength dependent magnification, and as a result, the relative size of objects vary based on the rendering color. In addition, this leads to color changes in the image due to mixing with neighboring pixels, which impacts image quality. The test results for the Oculus Go show promise for characterizing TCA across different HMDs. 


\section{INTRODUCTION}

Advances in display technology, computing capabilities, and optical fabrication have lead to a revived effort in the development of virtual (VR) and augmented reality (AR). While the majority of this new momentum has been mo- tivated by the entertainment industry for consumer device applications, these advances are also highly relevant for more professional applications, such as displaying and visualizing 3D medical data. Some of the medical applications currently being explored included displaying MRI segmentation [1], therapeutic uses [2], and training [3].

While AR and VR open new frontiers for medical ap- plications, it also raises unanswered questions concerning the performance of the devices. An important aspect of the performance are the optical aberrations, which alter the visual representation of the input data. One example is transverse chromatic aberration (TCA), which is a color dependent magnification [4]. Therefore, TCA could present a significant challenge for accurately rendering $3 \mathrm{D}$ segmentation data. Here we demonstrate a digital test pattern and setup to characterize the transverse chromatic aberration (TCA) of a device. We tested our method on the Oculus Go and found noticeable TCA for angles above approximately $6^{\circ}$.

\section{METHODS}

Figure 1 shows a sketch and picture of the experimental setup for characterizing a VR HMD. An achromatic lens ( $f=25 \mathrm{~mm}$ ) focused the light from the HMD onto a monochrome CCD camera. The optical setup has rotation and translation degrees of freedom, as shown. In this illustration, a single offaxis white pixel is illuminating the measurement setup. As the light passes through the lens in the VR HMD, the RGB components are focused to different lateral locations on the detection CCD. The displacement on the CCD and the image of the HMD can be used to provide a measure of the TCA.
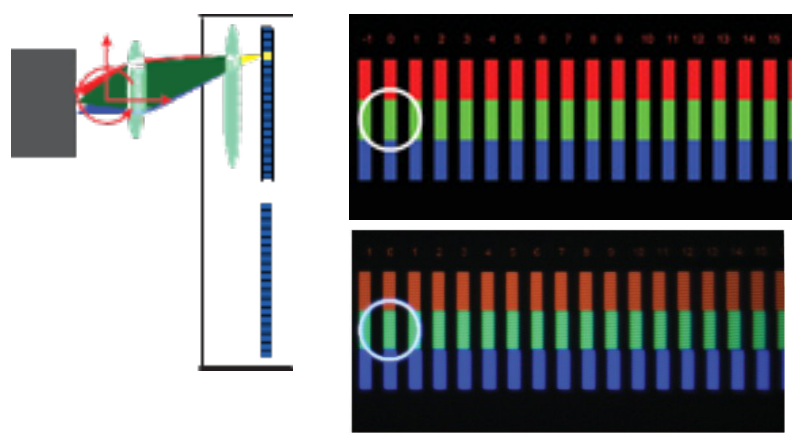

Fig. 1. (a) Sketch of a virtual reality headmounted display (VR HMD) with transverse chromatic aberration (TCA). (b) TCA test pattern sent to Oculus Go, where the white circle is the center of the field of view. (c) Resulting monochrome image acquired using the setup shown in (a).

The TCA was tested by displaying the test pattern, shown in Figure $1 \mathrm{~b}$, on the of the Oculus Go and the resulting image is shown in Figure 1 c. As can be seen in this image, the test pattern bars are blurred and displaced on the extrema of the field of view. This effect is more significant for the blue bars (top row) than for the red bars (second to bottom row), which is an indication of TCA in the system. 
Vol. 1, No. 1 - 2019

Frameless

\section{CONCLUSION}

These results demonstrate that our test pattern and optical setup provide a straight forward method for measuring the TCA of a HMD. While the presented results are only for the Oculus Go, this same approach is suitable for other devices and provides a convenient method for characterizing TCA in virtual reality devices. As the development of HMDs continues to increase as well as the applications for medicine, the necessity for suitable testing methods will continue increase.

\section{REFERENCES}

[1] D. Duncan, R. Garner, I. Zrantchev, T. Ard, B. Newman, A. Saslow, E. Wanserski, and A. W. Toga, "Using virtual reality to improve per- formance and user experience in manual correction of mri segmentation errors by non-experts," Journal of digital imaging, pp. 1-8, 2018.

[2] M. M. North and S. M. North, "Virtual reality therapy," in Computerassisted and web-based innovations in psychology, special education, and health. Elsevier, 2016, pp. 141-156.

[3] G. S. Ruthenbeck and K. J. Reynolds, "Virtual reality for medical training: the state-of-the-art," Journal of Simulation, vol. 9, no. 1, pp. 16-26, 2015.

[4] W. J. Smith, Modern optical engineering. Tata McGraw-Hill Education, 1966. 